\title{
Resilience: is it time for a rethink?
}

\begin{abstract}
We often associate resilience with burnout and link this with an intention to leave in the nursing and midwifery profession. Some believe that nursing and midwifery students are not resilient and that this impacts the development of coping strategies which might be adopted to manage burnout. Students themselves describe their studies as stressful and many report high levels of anxiety. This can impact on the rate of course attrition and the health and wellbeing of students. Higher education providers
\end{abstract}

are exploring ways of embedding wellbeing strategies which they hope will impact resilience, which will then help these students practice in challenging situations. In addition to considering the ways we teach and assist students, there is a growing focus on improving work cultures that move from individual notions of resilience towards the development of resilient workplaces, allowing nurses and midwives to flourish.
When considering the meaning of resilience and what it means to be resilient, we are presented with a myriad of definitions and inherent complexity in trying to form a definition. Resilience, in broad terms, is a process of adapting well in the face of adversity, stress, or tragedy, and can be described as a character trait that individuals use to develop strategies in order to bounce back in difficult situations.

Burnout is often linked with resilience, occurring when an individual can no longer manage the effects of stress, ${ }^{1}$ and is one of the issues linked with an intention to leave among nurses and midwives. A growing dissatisfaction with the professions is often associated with a lack of resilience, particularly in early career nurse and midwives. ${ }^{2}$

\section{The nursing and midwifery professions are often} acknowledged as being stressful and emotionally demanding. The work demands care is provided to people who are often at some of the most challenging times of their lives, and this impacts on the individuals who seek to provide them with that care. These same challenges also impact nursing and midwifery students. Stress and low resilience have been found in nursing students and the subsequent impact of latent burnout is not clear. ${ }^{3}$ Health professional students, including nursing and midwifery students, often perceive their education to be stressful and report increasing levels of anxiety, fatigue and a lack of motivation. ${ }^{4}$ This is often linked to high levels of course attrition, and the health and wellbeing of students is a common discussion point among higher education providers.

There is growing recognition that nurses and midwives need assistance in developing the knowledge and skills to work in challenging and complex environments, and resilience is increasingly viewed as a core graduate capability. 5 Promoting resilience in students through targeted educational interventions might be one method to address workforce burnout. A critical review published in 2009 recommended that all health professional programs include a focus on resiliency. ${ }^{6}$ This review recommended that higher educational providers provide opportunities that foster positive role modelling, mentoring, and coaching. In addition, the review reinforced the importance of instilling critical reflective and critical thinking skills in curriculum that assists students to foster altruism.

In the last ten years since this review was published, there has been minimal attention to the development of strategies or interventions designed to enhance resilience. This is surprising, given the obvious correlation between stress, burnout, and intention to leave the profession. ${ }^{4}$ One of the barriers towards improving resilience in the student cohorts, is the lack of a shared definition, common theory or model of resilience. This makes it 'difficult to develop quality measures of resilience',4(P70) and to determine whether any strategies we develop are effective.

Resources that assist students to engage in identity building, capacity and strengths development, and leadership, are well placed to facilitate resilience, and when used have shown a shift towards proactive promotion of resilience. ${ }^{4}$ However, many of these resources and their delivery methods were not well evaluated. Only one research study described a whole curriculum approach suggesting that while there are pockets of good work on developing resilience in the student population, further work must be achieved if real differences are to be made. ${ }^{7}$

Some educational providers have adopted approaches to foster student resilience including mentoring and coaching through linkage with industry providers. Some providers partnered final year students with those in earlier years, 
assisting students to share experiences, and providing avenues for final year students to provide tips which facilitated reflection and constructive thinking. ${ }^{8}$ Mindfulness based stress reduction and conflict resolution training have also been found to positively impact on resiliency, as have training programs that promote self-regulation, self-care and interpersonal relationships. ${ }^{1}$ Experiential learning, critical reflection and creativity have also been identified as useful methods for promoting resilience. ${ }^{5}$

While local strategics and resources have been used, it is clear that higher education providers must engage in transformative educational methods. Exploring how we teach, rather than what we teach is necessary if we are to facilitate the development of skills needed to promote resilience.

There is growing recognition of the need to move from individual notions of resilience towards the development of resilient workplaces in order for nurses and midwives to flourish. The impact of workplace culture and support is increasingly thought to impact on individual levels of resilience. For example, individuals who may have previously been 'resilient', may be susceptible to stress and burnout if the workplace environment is not supportive. Workplaces that support staff and students to have a sense of purpose, value person-centredness, and support the development of competencies and autonomy, are thought to support flourishing and reduce burnout. ${ }^{9}$

More recently, a qualitative study of community pharmacists highlighted that during the early stages of the COVID-19 pandemic, staff working in community pharmacies that invested in supportive actions such as changing shift length and providing sustenance were more able to respond and adapt to the stressful situation of working during a global pandemic..$^{10}$ There are lessons here for how we support students through clinical practice placements. Throughout the pandemic education providers have worked in tandem with health care providers to ensure the safety and support of students on placements while recognising the unique stresses that the clinical environment has been under.

\section{Professor Tracey Moroney}

Faculty of Science, Medicine and Health, University of Wollongong, New South Wales, Australia

\section{Professor Karen Strickland}

School of Nursing Midwifery and Public Health, University of Canberra, Australian Capital Territory, Australia

\section{REFERENCES}

1. Brown $S$, Whichello R, Price $S$. The impact of resiliency on nurse burnout: an integrative literature review. Medsurg Nurs. 2018; 27(6): 349-8.

2. Concilio L, Lockhart J, Oermann M, Kronk R, Schreiber J. Newly licensed nurse resiliency and interventions to promote resiliency in the first year of hire: an integrative review. J Contin Educ Nurs. 2019; 50(4): 153-61.

3. Sam $P$, Lee $P$. Do stress and resilience among undergraduate nursing students exist? Int J Nurs Educ. 2020; 12(1): 146-9.

4. Sanderson B, Brewer M. What do we know about student resilience in health professional education? A scoping review of the literature. Nurse Educ Today. 2017; (58): 65-71.

5. McGowan J, Murray K. Exploring resilience in nursing and midwifery students: a literature review. J Adv Nurs. 2016; 72(10): 2272-83.

6. McAllister M, McKinnon J. The importance of teaching and learning resilience in the health disciplines: a critical review of the literature. Nurse Educ Today. 2009; 29(4): 371-9.

7. Slavin S, Schindler D, Chibnall J. Medical student mental health 3.0: improving student wellness through curricular changes. Acad Med. 2014; 89(4): 573 - 7.

8. Beckman $\mathrm{H}$. The role of medical culture in the journey to resilience. Acad Med. 2015; 90(6): 710-2

9. Dewing J, McCormack B. Editorial: Tell me, how do you define person-centredness? J Clin Nurs. 2017; 26: 2509-10.

10. Austin Z, Gregory P. Resilience in the time of pandemic: the experience of community pharmacists during COVID-19. Res Soc Admin Pharm. 2021; 17(1): 1867-75. 